\title{
PEPPo: Using a polarized electron beam to produce polarized positrons
}

\author{
J. Grames ${ }^{1}$, on behalf of the PEPPo Collaboration \\ Thomas Jefferson National Accelerator Facility \\ 600 Kelvin Drive Suite 3, Newport News, VA 23606, USA \\ E-mail: gramesejlab.org
}

Polarized positron beams are identified as either an essential or significant ingredient for the experimental program at present or next generation lepton accelerators (JLab, Super KEK B, ILC, CLIC). A proof-of-principle experiment for a new method to produce polarized positrons has recently been performed at the Continuous Electron Beam Accelerator Facility at Jefferson Lab. The PEPPo (Polarized Electrons for Polarized Positrons) concept relies on the production of polarized electron/positron pairs from the bremsstrahlung radiation of a longitudinally polarized electron beam interacting within a high $\mathrm{Z}$ conversion target. The experiment was performed at the injector of the CEBAF accelerator at Jefferson Lab and investigated the polarization transfer of an $8.2 \mathrm{MeV} / \mathrm{c}$ polarized electron beam to positrons produced in varying thickness tungsten production targets, and collected and measured in the range of 3.1 to 6.2 $\mathrm{MeV} / \mathrm{c}$. This technique potentially opens a new pathway for both high energy and thermal polarized positron beams. This presentation will discuss the PEPPo concept, the motivations of the experiment and the experimental results so far obtained.

XVth International Workshop on Polarized Sources, Targets, and Polarimetry September 9-13, 2013

Charlottesville, Virginia, USA

1

Corresponding author: grames@jlab.org 


\section{Introduction}

The interest of the Physics Community in polarized positrons has been expressed in numerous projects involving thermal to very high beam energies (see Ref. [1] for examples). While storage ring facilities can rely on the natural self-polarization build-up from the SokolovTernov effect [2], external beam or continuous wave facilities require new scenarios. These new schemes rely on the polarization transfer from circularly polarized photons interacting in a high $\mathrm{Z}$ target to produce longitudinally polarized $\mathrm{e}+\mathrm{e}-$ pairs via the pair creation process $[3,4]$. The technique used for the production of polarized photons distinguishes the different schemes.

Within the context of the International Linear Collider (ILC), two techniques were experimentally investigated: Compton backscattering of a polarized laser from a $1.3 \mathrm{GeV}$ electron beam [5], and the synchrotron radiation of a $46.6 \mathrm{GeV}$ electron beam travelling through a helical undulator [6]. Both techniques produced highly polarized positrons, demonstrating the efficiency of the pair production process for a polarized positron beam. However, they both require $\mathrm{GeV}$ electron beams and challenging technologies therefore increasing the scale of a potential positron facility.

A novel approach is developed at the Continuous Electron Beam Accelerator Facility (CEBAF) of JLab, that takes advantage of its high performance polarized electron source [7,8]. Circularly polarized photons originate here from the bremsstrahlung radiation of a few $\mathrm{MeV}$ longitudinally polarized electron beam interacting in a tungsten target. Longitudinally polarized $\mathrm{e}+\mathrm{e}-$ pairs are then produced within the same target from the pair creation induced by polarized photons. This technique was initially proposed $[9,10]$ in the context of the ILC project but was not pursued. The performances of polarized electron sources at that time might have been considered as a strong limitation. Nowadays, the bremsstralhung of (unpolarized) polarized electrons is commonly used for the generation of (linearly) circularly polarized photon beams. Together with the successful experimental investigations of the photon polarization transfer [5, 6], this novel technique that we identify as the Polarized Electrons for Polarized Positrons (PEPPo) concept should be viable. Additionally, involving a low energy high intensity electron driver [7] the PEPPo concept can potentially open acccess to low intensity polarized positrons for a wider Community.

\section{Experiment Setup}

The PEPPo experiment [11] was designed to evaluate and demonstrate the PEPPo concept for a polarized positron source. It involved the construction of a new beam line at the CEBAF injector where up to $8.25 \mathrm{MeV} / \mathrm{c}$ polarized electrons were transported towards a tungsten positron production target followed by a positron collection, selection, and characterization system built on parts of the SLAC E-166 experimental setup [12] reconfigured for PEPPo purposes.

\subsection{General Layout}

The CEBAF injector [13] constitutes a full scale continuous low energy polarized electron beam facility with variable energy, providing continuous electron beams up to $200 \mu \mathrm{A}$ 
intensities and $89 \%$ polarization, and equipped with the necessary beam characterization apparatus for the completion of the experiment. This comprises the control and monitoring of the beam polarization and intensity, the measurement of the electron beam energy with a dedicated spectrometer line, and the measurement of the electron beam polarization with a Mott polarimeter. The PEPPo experiment was developed on a new beam line branch section (Fig. 1) composed of four different regions. The first region is dedicated to the electron beam transport

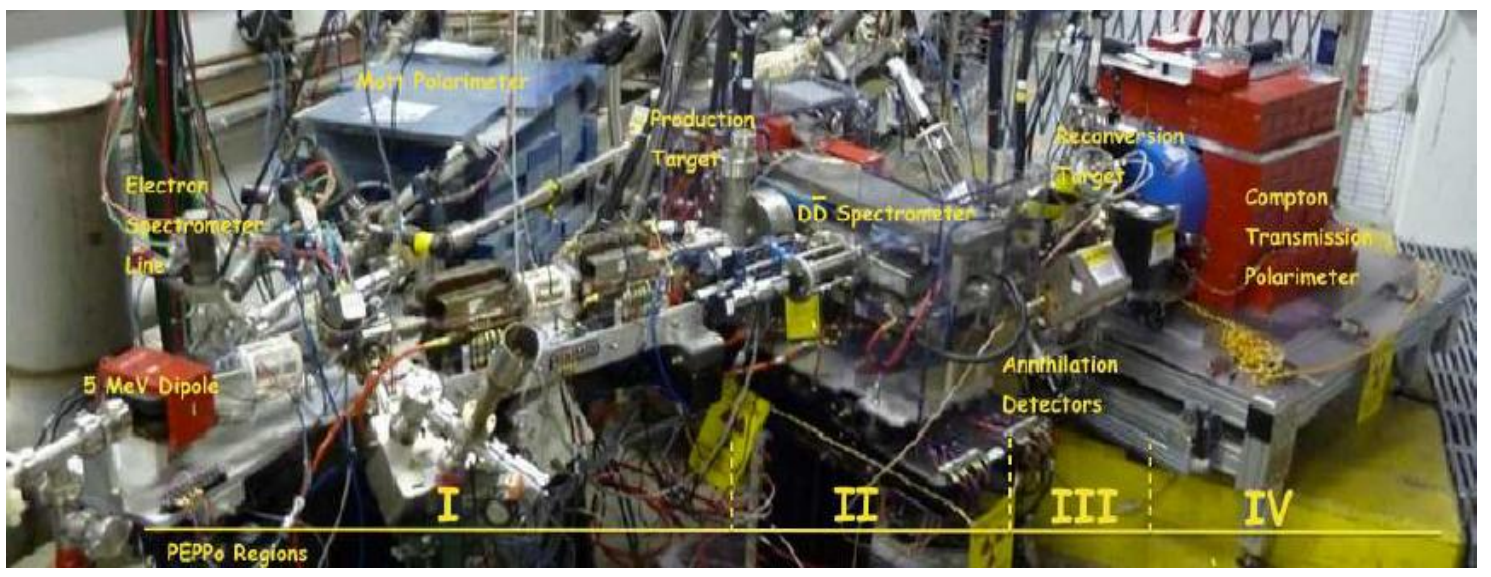

Figure 1. The PEPPo beam line, starting from the so-called $5 \mathrm{MeV}$ dipole branch and extending up to the Compton transmission polarimeter enclosed in a lead shielding housing.

to the positron production target (T1) and is equipped with active elements (quadrupole and corrector magnets to set beam size and position) and beam diagnostics (viewers and beam position monitors). The second region concerns the positron collection and selection made of a short focal length solenoid lens and a D D-bar spectrometer with $\pm 90^{\circ}$ bend dipoles. Additional beam diagnostic (viewer) and selection devices (adjustable collimator) complete instrumentation of this section. The third region is intended to optimize the positron transport to the Compton transmission polarimeter using a solenoid lens and corrector magnets, and comprises also several beam diagnostic (viewers, Faraday cup) and a specific $\mathrm{e}+\mathrm{e}-$ annhilation detector system designed to detect the presence of positrons at the exit of the spectrometer. The last region of the PEPPo apparatus consists of the electron and positron characterization device where the polarization of incoming particles is measured.

\subsection{Compton Transmission Polarimetry}

PEPPo polarimetry is based on the sensitivity of the Compton scattering process to the polarization of incoming photons. At the exit of the vacuum beam line, incoming polarized electrons or positrons are first converted into polarized photons by bremsstrahlung (and annihilation for positrons) radiation inside a $2 \mathrm{~mm} \mathrm{~W}$ target (T2). These photons next interact with a $7.5 \mathrm{~cm}$ long polarized iron target, where photon absorption inside the iron target depends upon the relative orientation of the iron target polarization with respect to the photon. The transmitted photons are detected in a segmented calorimeter composed of a $3 \times 3$ CsI crystal array. By measuring the number or energy of photons transmitted through the iron target for opposite target or photon polarization, one obtains a measurement of the initial photon polarization. The experimental asymmetry is measured with respect to the helicity flip of the 
CEBAF electron beam, and can be written $\mathrm{A}_{\mathrm{T}}=\mathrm{P}_{\mathrm{e} \pm} \cdot \mathrm{P}_{\mathrm{T}} \cdot \mathrm{A}_{\mathrm{e} \pm}$, where $\mathrm{P}_{\mathrm{e} \pm}$ is the electron/positron polarization, $\mathrm{P}_{\mathrm{T}}$ is the iron target polarization determined from the knowledge of the polarizing magnetic field [14], and $A_{e \pm}$ is the analyzing power of the detection system [15]. Knowing the initial beam polarization, the experimental asymmetry $A_{T}$ measures the analyzing power of the detector (electron data) while knowing the analyzing power, $\mathrm{A}_{\mathrm{T}}$ measures the polarization of the incoming beam (positron data).

\section{Data Taking}

\subsection{Experimental Method}

The PEPPo experiment took advantage of the well-defined CEBAF electron beam for a full calibration of the apparatus. The gradients of two superconducting radio-frequency cavities were first calibrated with the electron spectrometer providing $~ 0.5 \%$ measurement of the beam momentum. The transport of this electron beam through the PEPPo line calibrates the D D-bar spectrometer versus electron momentum. Inserting a production target at $\mathrm{T} 1(0.1$ or $1.0 \mathrm{~mm} \mathrm{~W})$ and optimizing the collection of the degraded electron beam with the Faraday cup at the end of the vacuum section of the PEPPo line calibrates the solenoid collection settings at a given spectrometer momentum. The experimental determination of the optical properties of the PEPPo line is in good agreement with beam transport simulations based on the magnetic field mapping of each element prior to installation in the injector tunnel. Switching between electron and positron measurements is simply achieved by reversing the polarity of the spectrometer.

Measurement of the Compton asymmetry using the electron beam allows the experimental determination of the electron analyzing power of the Compton transmission polarimeter [15]. This step is intended to benchmark a GEANT4 model of the polarimeter from which we later simulate the positron analyzing power, therefore electron analyzing power measurements were performed at the same four beam energies of which positrons were collected. Important to this model, electrons and positrons differ by the positron annihilation process. Within the PEPPo calibration procedure, the control of this mechanism is obtained indirectly from interactions of secondary positrons created by multiple interactions of incident electrons and bremsstrahlung photons.

\subsection{Polarization Transfer Data}

The measurement of the longitudinal polarization transfer from electrons to positrons has been performed with a $8.25 \mathrm{MeV} / \mathrm{c}$ incident electron beam on two $\mathrm{W}$ targets with different thicknesses (Tab. 1) using an electron beam polarization of $83.4 \%$ as measured by a Mott polarimeter. The collection of positrons was done using a momentum scan of the positron polarization spectra over the range 3.2-6.2 MeV/c. The momentum scan is a core measurement of the PEPPo experiment, intended to demonstrate the reliability of the PEPPo concept. The target thickness dependence is also an important measurement expected to show the sensitivity to the details of the physics at work in this concept: particularly, distinguishing between ultrarelativistic [3] and finite lepton mass [4] descriptions of the polarization transfer in bremsstrahlung and pair production processes, and possibly investigating the eventual contribution of virtual pair production. 
Experimental data were collected within two separate sets with initial (Set 1) and optimized (Set 2) shielding configurations, as well as beam intensities and analysis procedures

\begin{tabular}{cccc}
\hline $\begin{array}{c}\mathbf{P}_{\mathrm{e}+} \\
(\mathbf{M e V} / \mathbf{c})\end{array}$ & $\begin{array}{c}\mathbf{I}_{\mathrm{e}-} @ \mathbf{T 1}(\mathbf{n A}) \\
\text { Set } \mathbf{1}\end{array}$ & $\begin{array}{c}\mathbf{I}_{\mathrm{e}-} @ \mathbf{T 1}(\mathbf{n A}) \\
\text { Set 2 }\end{array}$ & $\begin{array}{c}\text { Target } \\
(\mathbf{m m})\end{array}$ \\
\hline $\mathbf{3 . 2}$ & 380 & 120 & 1.0 \\
$\mathbf{4 . 2}$ & 25 & 130 & 1.0 \\
$\mathbf{5 . 5}$ & 95 & 200 & 1.0 \\
$\mathbf{6 . 3}$ & 380 & 620 & 1.0 \\
$\mathbf{3 . 2}$ & - & 380 & 0.1 \\
\hline
\end{tabular}

Table 1. Positron measurements.

involving different analyzing power. Each positron momentum point corresponds to a collection of a large number of runs where the experimental asymmetry is measured with respect to the initial electron beam helicity for a given status of the electron source laser polarization $( \pm)$ and of the iron target polarization orientation $( \pm)$. In order to control and minimize systematic effects, experimental data have been acquired for each configuration following a random sequence. Systematics is further reduced by the helicity structure of the beam which follows a quartet pattern where helicity is flipped at a $30 \mathrm{~Hz}$ frequency. The experiment trigger is built from the coincidence between a $1 \mathrm{~mm}$ thick scintillator placed prior $\mathrm{T} 2$ at the entrance of the polarimeter, and the central crystal of $3 \times 3$ detector array, leading to a dramatic reduction of the neutral background. Each event is tagged by the beam helicity information reported in a delayed mode, electronically isolating the real time helicity and detector signals.

At the time of this Workshop the simulated GEANT4 model of the positron analyzing power is not yet completed. However, it is possible to obtain a preliminary indication of the positron polarization by extrapolating from the measured electron analyzing power. To do this the energy-integrated positron asymmetry for each run is computed by summing the energies of

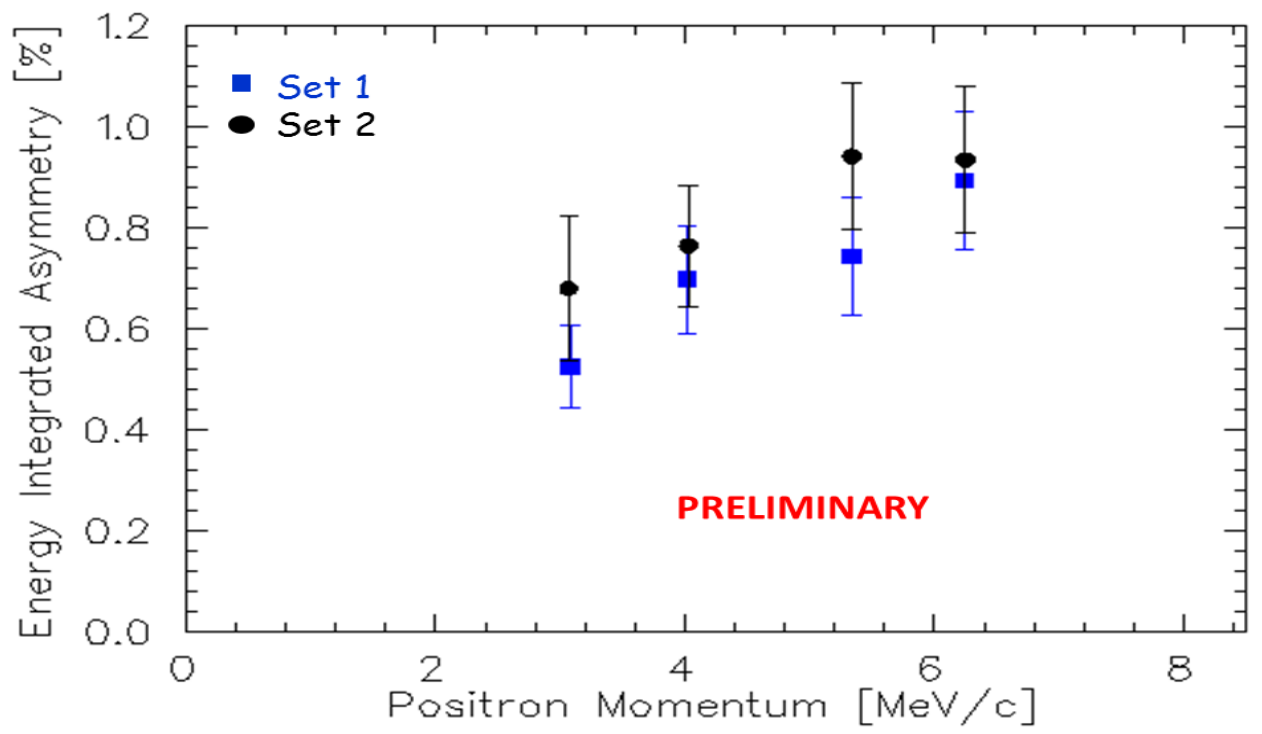

Figure 2. Statistically averaged energy integrated asymmetries of collected positrons. 
the detected photons above the annihilation threshold $(>511 \mathrm{keV})$ up to the bremsstrahlung end point (positron energy $-\mathrm{m}_{\mathrm{e}+}$ ). The run averaged asymmetries using the $1 \mathrm{~mm} \mathrm{~W}$ target are computed and shown in Fig. 2 as a function of momentum. As stated above the connection to positron polarization is made using the measured electron energy-integrated analyzing power. This is accomplished by applying the measured electron analyzing power at each energy with a correction which estimates the relative size of the electron and positron analyzing power. The size of the correction ranges from 10-40\% over the momenta studied and is estimated from a similar comparison using a Compton transmission polarimeter over a comparable energy range [12]. The positron polarization computed by this method is shown in Fig. 3 and includes a large $20 \%$ uncertainty to the correction. In this preliminary analysis the results of Fig. 3 are consistent with positron polarization exceeding $50 \%$, as expected for this range of measured positron momenta.

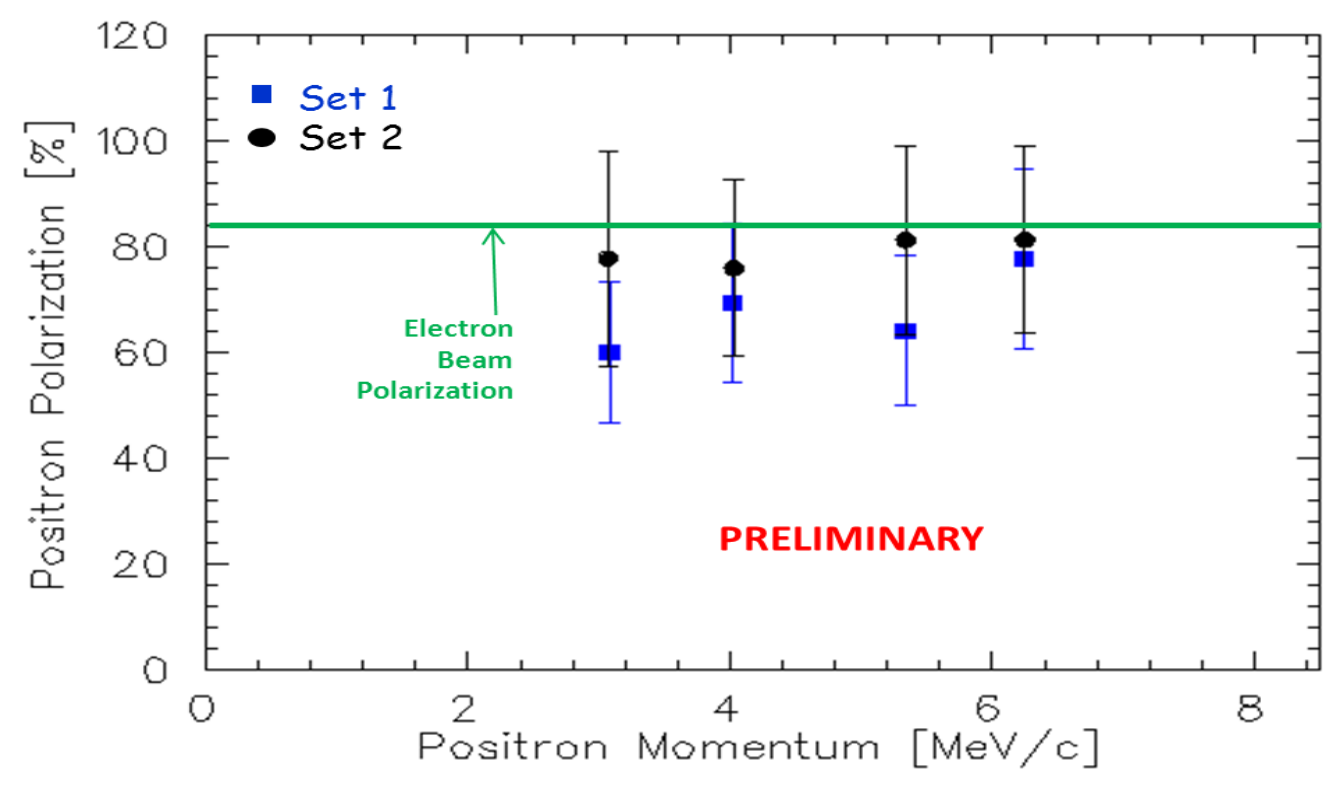

Figure 2. Statistically averaged energy integrated asymmetries of collected positrons.

\section{Conclusion}

The PEPPo experiment at JLab did measure the longitudinal polarization transfer from 8.25 MeV/c electrons to positrons of different momentum produced via polarized bremsstrahlung and pair production in a tungsten target. The positron polarization was measured with a Compton transmission polarimeter. Significant experimental asymmetries are reported from preliminary analysis which indicates high positron polarization, well above $50 \%$. The analysis is progressing and we hope to provide final results of the positron polarization with uncertainty less than $10 \%$ next year.

\section{Acknowledgment}

We are deeply grateful to the SLAC E-166 Collaboration, particularly K. Laihem, K. McDonald, S. Riemann, A. Schalicke, P. Schuler, J. Sheppard and A. Stahl for loan of fundamental equipment parts and support in GEANT4 modeling. We also thank N. Smirnov for 
coordinating delivery of critical hardware. This work was supported in part by the U.S. Department of Energy, the French Centre National de la Recherche Scientifique and the International Linear Collider project. Jefferson Science Associates operates the Thomas Jefferson National Accelerator Facility under DOE contract DE-AC05-06OR23177.

\section{References}

[1] Proceedings of the International Workshop on Positrons at Jefferson Lab, Edts. L. Elouadrhiri, T.A. Forest, J. Grames, W. Melnitchouk, E. Voutier, AIP Conf. Proc. 1160 (2009).

[2] A.A. Sokolov, I.M. Ternov, Sov. Phys. Dokl. 8 (1964) 1203.

[3] H. Olsen, L. Maximon, Phys. Rev. 114 (1959) 887.

[4] E.A. Kuraev, Y.M. Bystritskiy, M. Shatnev, E. Tomasi Gustafsson, Phys. Rev. C 81 (2010) 055208 .

[5] T. Omori et al., Phys. Rev. Lett. 96 (2006) 114801.

[6] G. Alexander et al., Phys. Rev. Lett. 108 (2008) 210801.

[7] J. Grames et al., THPMS064 (2007).

[8] P.A. Adderley et al., Phys. Rev. ST Accel. Beams 13 (2010) 010101.

[9] E.G. Bessonov, A.A. Mikhailichenko, THP071L (1996).

[10] A.P. Potylitsin, Nucl. Inst. Meth. A 398 (1997) 395.

[11] J. Grames, E. Voutier et al., JLab Experiment E12-11-105 (2012).

[12] G. Alexander et al., Nucl. Inst. Meth. A 610 (2009) 451.

[13] R. Kazimi et al., TUPLT164 (2004).

[14] O. Dadoun, E. Froidefond, E. Voutier, IPAC2013, MOPWA079 (2013).

[15] A. Adeyemi, E. Voutier, IPAC2013, MOPWA078 (2013). 